\title{
Liver diffusion-weighted MR imaging: the tower of Babel?
}

\author{
Boris Guiu • Jean-Pierre Cercueil
}

Received: 4 September 2010 / Accepted: 11 October 2010 /Published online: 26 November 2010

(C) European Society of Radiology 2010

\begin{abstract}
There is a growing amount of literature regarding diffusion-weighted imaging (DWI) of the liver. The apparent diffusion coefficient (ADC) was introduced in 1986 and is used extensively in studies. However, methods for calculating ADC vary considerably and the value of the ADC strongly depends on the $b$ values chosen for its calculation. Indeed, the ADC incorporates the effects of both diffusion and perfusion, which can vary independently. Since signal attenuation as a function of $b$ follows a bi-exponential pattern, other diffusion/perfusion coefficients can be calculated using DWI, and these may provide more meaningful measurements than the ADC. The absence of standardization for both the terminology and the methodology in DWI of the liver makes it difficult for readers to understand the technique used and strongly limits comparisons between studies. Here, we review the main principles of DWI of the liver, the limits of the ADC, and the exciting capabilities of multi-parametric DWI. We also insisted on the need for a common language for DWI of the liver.
\end{abstract}

Keywords Abdomen - Magnetic resonance imaging · ADC $\cdot$ Perfusion $\cdot$ Diffusion

B. Guiu · J.-P. Cercueil

University of Burgundy, INSERM U866,

BP 87900, 21079 Dijon, France

B. Guiu $(\bowtie) \cdot$ J.-P. Cercueil $(\bowtie)$

Department of Radiology, CHU (University Hospital),

2 boulevard Maréchal de Lattre de Tassigny,

BP 77908, 21079 Dijon, France

e-mail: Boris.guiu@chu-dijon.fr

J.-P. Cercueil

e-mail: Jean-pierre.cercueil@chu-dijon.fr
When reading papers in the gastrointestinal imaging section of European Radiology, we discovered that diffusionweighted imaging (DWI) of the liver appears to have been one of the hottest topics over the past 18 months. Indeed, we found at least 7 original papers $[1-4,10,12,13]$ dealing with DWI of the liver, each of which focused on different clinical applications ranging from the detection of hepatocellular carcinoma $[1,12]$ to the diagnosis of liver metastases $[1,4,10]$, the prediction of tumor response $[3,13]$ or the evaluation of liver steatosis [2].

These various applications are in keeping with the major capabilities of diffusion-weighted sequences to capture information regarding the molecular diffusion of water, which can be either restricted or enhanced depending on the pathology. The apparent diffusion coefficient (ADC) was introduced in 1986 [8] and its use is widely reported in the literature. Though software included in MR workstations can often perform mono-exponential regression with $>2 b$ values, the $\mathrm{ADC}$ is usually calculated by mono-exponential regression using two $b$ values with the formula:

$\mathrm{ADC}=\log _{\mathrm{e}}\left(\mathrm{S}_{0} / \mathrm{S}_{1}\right) /\left(b_{1}-b_{0}\right)$

The optimal $b$ values used for DWI remain unclear, which explains why methods for calculating the ADC vary considerably from one study to another. Furthermore, the ADC incorporates the effects of both diffusion and perfusion [14], which can vary independently. The main drawback is that ADC values depend on both the method used to calculate them and the chosen values for $b$. This considerably complicates the interpretation of the data.

As detailed below, the variability of the ADC can be explained by the use of a mono-exponential model, whereas signal attenuation is bi-exponential with DWI. Applying a mono-exponential fit to bi-exponential signal attenuation 
could be considered bad science and may remind us of the famous problem of squaring the circle. In addition to this, the huge variety in the terminology used in publications makes descriptions more difficult to understand and highlights the need for a common language, as was the case in the biblical account of the tower of Babel. Finally, other diffusion/perfusion coefficients can now be calculated using DWI, and these may provide more meaningful measurements than the ADC. Let us take stock of the situation concerning DWI of the liver.

\section{IVIM theory, ADC and other diffusion/perfusion coefficients}

The term intravoxel incoherent motion (IVIM) introduced by Le Bihan et al. several years ago [7] reflects the random microscopic translations that occur in voxels on MR images of either intracellular or extracellular water molecules and the microcirculation of blood, since the capillary network is organized pseudorandomly at the voxel level [14]. Since the liver has an isotropic structure, liver DWI is routinely estimated by using tridirectional diffusion gradients (along the 3 axes $\mathrm{x}, \mathrm{y}$ and $\mathrm{z}$ ) to calculate an average diffusionweighted image (called "trace") [11]. According to IVIM theory [7], signal attenuation as a function of $b$ is expressed by the following equation [6]:

$\mathrm{SI}=\mathrm{SI}_{0} *\left[(1-\mathrm{f}) * \exp \left(-b \times \mathrm{D}_{\text {slow }}\right)+\mathrm{f} * \exp \left(-b \times \mathrm{D}_{\text {fast }}\right)\right]$

where SI is the signal intensity at the given $b$ value, $\mathrm{f}$ represents the perfusion fraction (i.e., fractional volume occupied in the voxel by flowing spins), $\mathrm{D}_{\text {slow }}$ (also called D) represents pure molecular diffusion and $\mathrm{D}_{\text {fast }}$ (also called $\mathrm{D}^{*}$ ) perfusion-related diffusion. $\mathrm{SI}_{0}$ is proportional to $\exp (-\mathrm{TE} / \mathrm{T} 2)$, which explains why DWI performed with $b=0 \mathrm{~s} / \mathrm{mm}^{2}$ corresponds to a T2-weighted sequence. This should not be forgotten when optimizing DWI sequences especially to preserve an adequate signal-to-noise ratio (SNR) with high $b$ values (meaning that if $\mathrm{T} 2$ is short or TE is long, the SNR will be very low at high $b$ values). In a normal liver, the $D_{\text {slow }}$ value is about $1.3 \times 10^{-3} \mathrm{~mm}^{2} / \mathrm{s}$, the $D_{\text {fast }}$ value is about 100 times higher and $\mathrm{f}$ varies between $25 \%$ and $30 \%$ (Fig. 1). Given the relative values of $\mathrm{D}_{\text {slow }}$ and $\mathrm{D}_{\text {fast }}$, the signal from vessels with rapid flow disappears quickly as the $b$ value increases [5], which

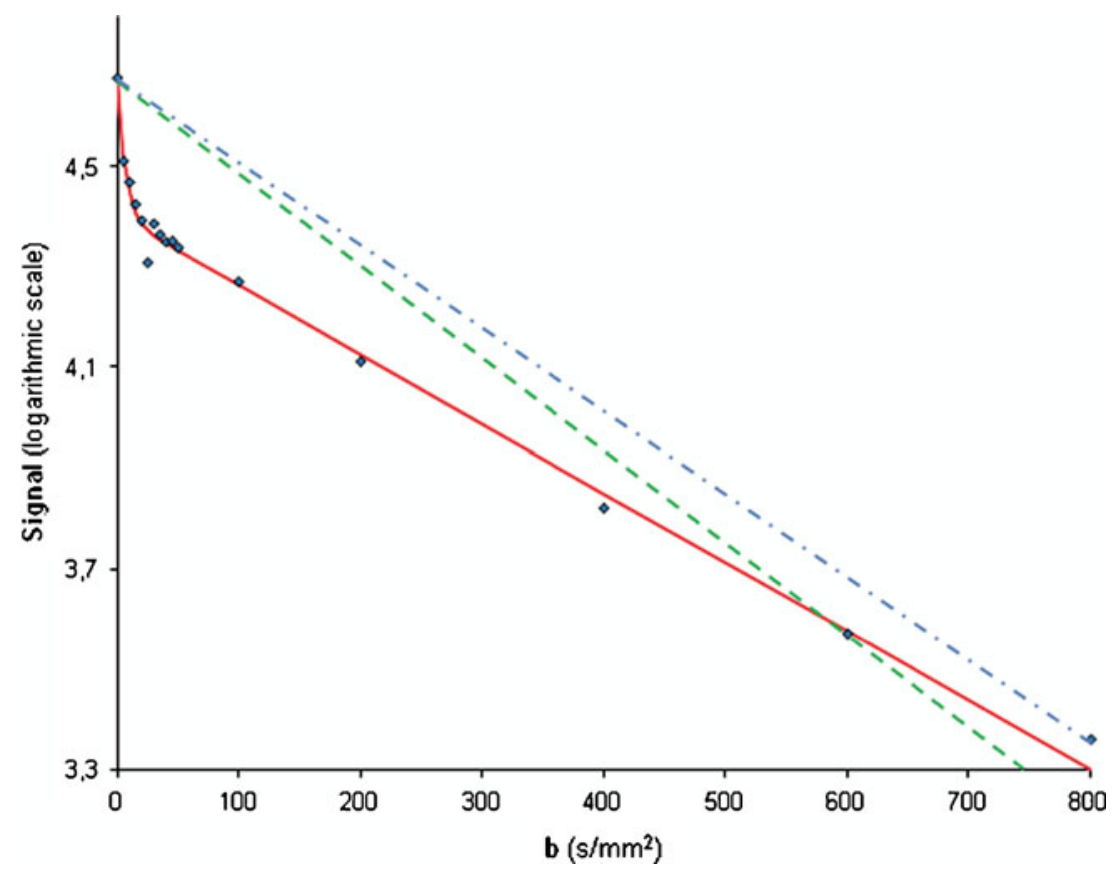

Fig. 1 Evolution of the signal measured using a diffusion-weighted sequence with $16 b$ values $(0,5,10,15,20,25,30,35,40,45,50$, $100,200,400,600,800 * 10^{-3} \mathrm{~s} / \mathrm{mm}^{2}$ ) in a 43 -year-old man with normal liver. (Siemens Trio Tim WIP, Erlangen, Germany). Points: recorded values; red line: bi exponential regression $\left(\mathrm{D}_{\text {slow }}=1.38 \times\right.$ $\left.10^{-3} \mathrm{~mm}^{2} / \mathrm{s}, D_{\text {fast }}=160 \times 10^{-3} \mathrm{~mm}^{2} / \mathrm{s}, \mathrm{F}=0.24\right)$; dashed blue line: 2 point mono-exponential regression $\left(b=0,800 \mathrm{~s} / \mathrm{mm}^{2}\right)$ for ADC calculation, dash-point green line: 2-point mono-exponential regression $\left(b=0,600 \mathrm{~s} / \mathrm{mm}^{2}\right)$ for ADC calculation. In a semi-logarithmic projection, a mono-exponential curve should give a straight line, whose slope is the ADC. Here, a bi-exponential model provides an excellent fit. The first part $\left(0<\mathrm{b}<50 \mathrm{~s} / \mathrm{mm}^{2}\right)$ of the fitting curve represents both pure molecular diffusion and perfusion-related diffusion, whereas the second part $\left(>50 \mathrm{~s} / \mathrm{mm}^{2}\right)$ reflects mostly pure molecular diffusion. The ADC (i.e. the slope) varies considerably (and is overestimated) when $b=0 \mathrm{~s} / \mathrm{mm} 2$ is used to calculate it, because the effect of perfusion is also incorporated in the calculation 
Table 1 ADC values calculated from the 16- $b$ diffusion-weighted sequence of Fig. 1, using a 2-point monoexponential regression according to the $\mathrm{b}$ values used

\begin{tabular}{lllll}
\hline \multicolumn{1}{c}{ First $b$ value } & 0 & 50 & 100 & 200 \\
\hline $\begin{array}{l}\text { Second } b \text { value } \\
200\end{array}$ & 2.83 & 1.51 & 1.59 & \\
400 & 2.14 & 1.48 & 1.50 & 1.45 \\
600 & 1.84 & 1.40 & 1.40 & 1.35 \\
800 & 1.65 & 1.30 & 1.30 & 1.25 \\
\hline
\end{tabular}

The ADC varies considerably $(1.65-2.83 * 10-3 \mathrm{~mm} 2 / \mathrm{s})$ and is overestimated when $b=0 \mathrm{~s} / \mathrm{mm} 2$ is used to calculate it, because the effect of perfusion is incorporated in the calculation. With $a$ fist $b$ value $>50 * 10^{-3} \mathrm{~s} / \mathrm{mm}^{2}$, we obtain more reliable results.

When a 6-point logarithmic regression is used $(b=50,100,200,400,600$, $800)$, the $\mathrm{ADC}=1.37 * 10-3 \mathrm{~mm} 2 / \mathrm{s}$ whereas, the $\mathrm{ADC}=1.36 * 10-3 \mathrm{~mm} 2 / \mathrm{s}$ with a 5-point logarithmic regression $(b=100,200,400,600,800)$. explains the black-blood images obtained at very low $b$ values. The value of the ADC strongly depends on the $b$ values chosen for its calculation. To show this, Table 1 contains ADC values calculated using a 2-point monoexponential regression according to the $b$ values used: the ADC varies considerably $\left(1.65-2.83 \times 10^{-3} \mathrm{~mm}^{2} / \mathrm{s}\right)$ and is overestimated when $b=0 \mathrm{~s} / \mathrm{mm}^{2}$ is used to calculate it, because the effect of perfusion is also incorporated in the calculation.

Interestingly, the signal due to $\mathrm{D}_{\text {fast }}$ (microperfusion) is very close to 0 as soon as the value of $b$ exceeds $50 \times 10^{-3} \mathrm{~s} / \mathrm{mm}^{2}$. Therefore, for $b$ values $>50-100 \times 10^{-3} \mathrm{~s} / \mathrm{mm}^{2}$, signal attenuation can be considered mono-exponential. Thus, the ADC calculated with $b>50 \mathrm{~s} / \mathrm{mm} 2$ as the first value (and not $b=0$ ) is more reproducible (Table 1) and corresponds to calculating $\mathrm{D}_{\text {slow }}$.
Fig. 2 Hepatocellular carcinoma developed in a non-cirrhotic liver. Besides the classical T2weighted turbo spin-echo image (a) and the single-shot diffusionweighted spin echo-planar sequence obtained with $a b$ value of $600 \mathrm{~s} / \mathrm{mm} 2(\mathbf{b})$, parametric images were calculated (using ImageJ software) from the same diffusion-weighted sequence with 16 b values as in Fig. 1. On the $\mathrm{D}_{\text {slow }}$ cartography $(\mathbf{c})$, the HCC nodule exhibits moderately low signal intensity (i.e. moderately restricted pure molecular diffusion; mean $\mathrm{D}_{\text {slow }}$ for HCC: $0.94 \times 10^{-3} \mathrm{~mm}^{2} / \mathrm{s}$; mean $\mathrm{D}_{\text {slow }}$ for surrounding liver: $\left.1.08 \times 10^{-3} \mathrm{~mm}^{2} / \mathrm{s}\right)$. The $D_{\text {fast }}$ cartography $(\mathbf{d})$ is more noisy, but reveals low perfusionrelated diffusion for the $\mathrm{HCC}$ nodule (mean $\mathrm{D}_{\text {fast }}$ for HCC: $29 \times 10^{-3} \mathrm{~mm}^{2} / \mathrm{s}$; mean $D_{\text {fast }}$ for surrounding liver: $92 \times$

$10^{-3} \mathrm{~mm}^{2} / \mathrm{s}$ ). Finally, the $\mathrm{f}$ cartography (e) demonstrates a very low perfusion fraction in the HCC (mean f for HCC: 0.06; mean $\mathrm{F}$ for surrounding liver: 0.32). Color-coded multiparametric image (f) provides immediate simultaneous visual evaluation of the 3 diffusion parameters
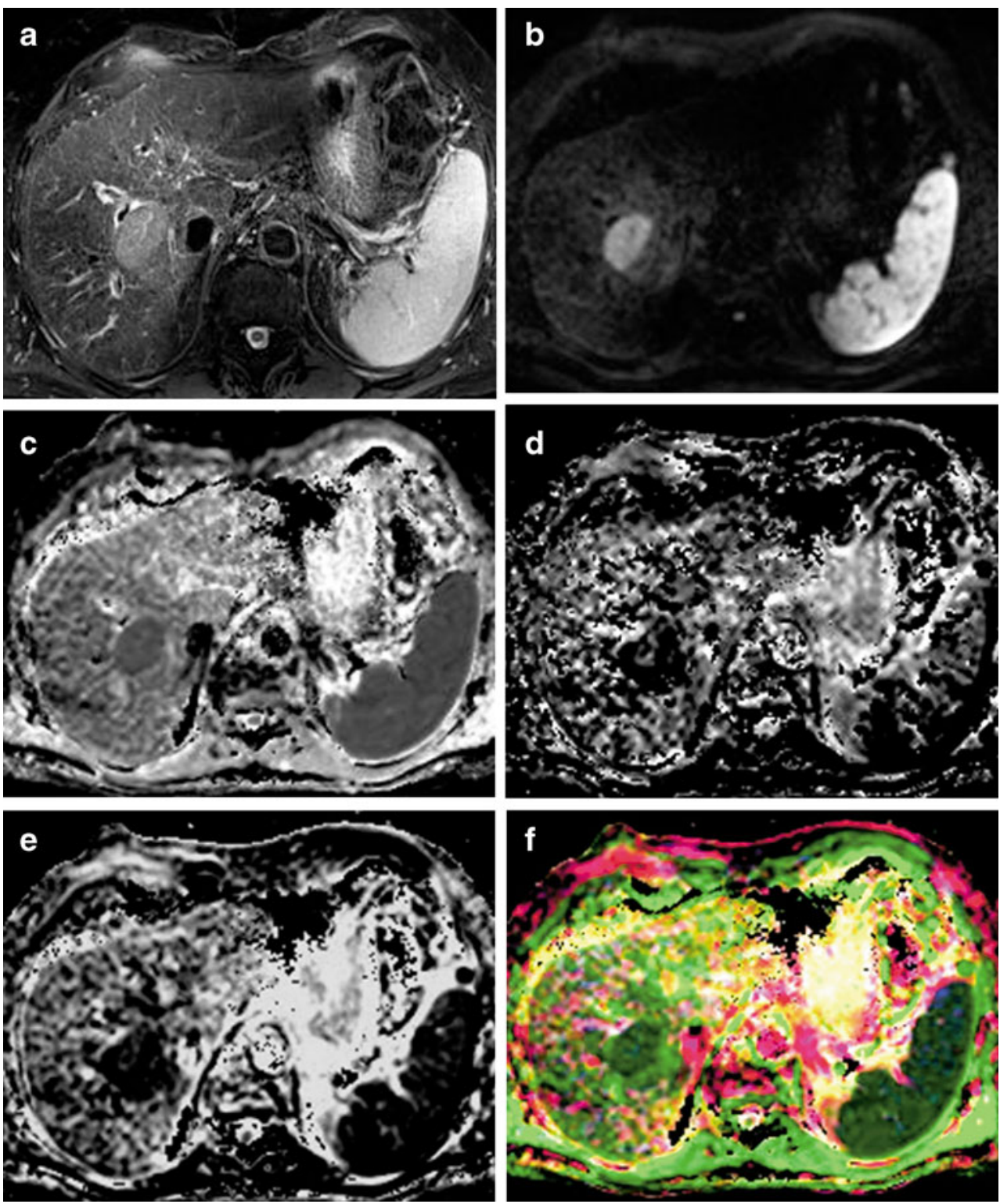
As shown in Table 1, substantial variations of ADC values persist even when $b$ values greater than $50 \mathrm{~s} / \mathrm{mm}^{2}$ are used. This can be mostly explained by the noise. The greater the $b$ value, the smaller the SNR. Even at $3 \mathrm{~T}$, with state-of-art gradient hardware, reduced echo times, increased number of acquisitions and respiratory triggering, SI of the liver is very low at $b$ values greater than $800 \mathrm{~s} / \mathrm{mm}^{2}$ thereby explaining why noise may contribute substantially to the signal and could influence the calculation of diffusion coefficients. Of course, calculation of the ADC (or other diffusion parameters) can be and should be performed using more than two $b$ values, thereby reducing the effect of noise, provided that the $b$ values are carefully chosen.

Bi-exponential fitting makes it possible to calculate $\mathrm{f}$ and $D_{\text {fast }}$, but very slow $b$ values (between 0 and $20 \mathrm{~s} / \mathrm{mm}^{2}$ ) are needed to model the first part of the curve correctly and thus to provide reliable results for $\mathrm{D}_{\text {fast }}$. Otherwise, its value may be greatly underestimated.

\section{Towards standardization...}

To make articles on the subject more easily understandable to readers, the terminology must be clear and standardized if possible. Obviously, we are not in a position to impose rules, but we would like to propose some channels for reflection. First, a precise description of the method used to calculate ADC should be reported in papers since ADC values are meaningful only when reported with the $b$ values used for their measurement, as stated above. Given the significant perfusion fraction in the liver, we suggest abandoning calculation of the ADC using $b=0 \mathrm{~s} / \mathrm{mm} 2$ and replacing it with $b>50-100 \mathrm{~s} / \mathrm{mm} 2$. For the sake of clarity, we propose that the $b$ values used to calculate the ADC be systematically included close to the term "ADC" in both the manuscript and the abstract of papers. For instance, if $b$ values of 50,200 and $400 \mathrm{~s} / \mathrm{mm} 2$ are used to calculate the $\mathrm{ADC}$, the ADC may be cited as "ADC $(b=50,200,400)$ ".

With IVIM DWI, other diffusion parameters than the ADC can be calculated [9]. Again, terminology is of major importance, especially so since, to our knowledge, no specific recommendations have been published. The " $\mathrm{D}$ " or " $\mathrm{D}_{\text {slow" }}$ coefficient was called pure molecular diffusion coefficient by le Bihan. This name is probably more understandable than the term "true diffusion coefficient" proposed by Yamada et al. [14] suggesting that a "false" diffusion coefficient may exist (perhaps ADC?). The abbreviation $\mathrm{D}_{\text {slow }}$ has the advantage of being clearer. The " $\mathrm{f}$ " coefficient unanimously designates the perfusion fraction. Finally, the name of the " $\mathrm{D}$ *" or " $\mathrm{D}_{\text {fast }}$ " coefficient is more varied, called either "pseudodiffusion" or "perfusion-related diffusion" or even the "fast-component of diffusion". In order to be as meaningful as possible, perfusion-related diffusion coefficient is probably the best term and " $\mathrm{D}_{\text {fast }}$ " the best suited abbreviation.

The determination of these coefficients $\left(D_{\text {slow }}, D_{\text {fast }}\right.$ and $\left.f\right)$ seems promising in diffuse liver disease and especially in cirrhosis in which $D_{\text {fast }}$ is significantly reduced [9]. The exploration of focal lesions using the same approach opens an immense and exciting field to explore (Fig. 2). Currently, even though it is technically challenging to calculate the 3 diffusion coefficients with a high level of reproducibility in focal lesions, further improvements and the optimization of hardware, sequences and signal analysis should considerably improve the reliability of the results in the future.

To conclude, there is no doubt that the literature regarding DWI of the liver will grow in the future. We strongly encourage manufacturers to implement multi-b diffusionweighted echo-planar sequences in MR systems. With such sequences, the possibilities of DWI would be considerably expanded opening an exciting door onto microscopic imaging of both perfusion and molecular diffusion. And wouldn't it be wonderful if we all spoke the same language!

\section{References}

1. Choi JS, Kim MJ, Choi JY, Park MS, Lim JS, Kim KW (2010) Diffusion-weighted MR imaging of liver on 3.0-Tesla system: effect of intravenous administration of gadoxetic acid disodium. Eur Radiol 20:1052-1060

2. d'Assignies G, Ruel M, Khiat A, Lepanto L, Chagnon M, Kauffmann C, Tang A, Gaboury L, Boulanger Y (2009) Noninvasive quantitation of human liver steatosis using magnetic resonance and bioassay methods. Eur Radiol 19:2033-2040

3. Dudeck O, Zeile M, Wybranski C, Schulmeister A, Fischbach F, Pech M, Wieners G, Ruhl R, Grosser O, Amthauer H, Ricke J (2010) Early prediction of anticancer effects with diffusionweighted MR imaging in patients with colorectal liver metastases following selective internal radiotherapy. Eur Radiol 20:2699-706

4. Hardie AD, Naik M, Hecht EM, Chandarana H, Mannelli L, Babb JS, Taouli B (2010) Diagnosis of liver metastases: value of diffusion-weighted MRI compared with gadolinium-enhanced MRI. Eur Radiol 20:1431-1441

5. Le Bihan D (2008) Intravoxel incoherent motion perfusion MR imaging: a wake-up call. Radiology 249:748-752

6. Le Bihan D (2007) The 'wet mind': water and functional neuroimaging. Phys Med Biol 52:R57-90

7. Le Bihan D, Breton E, Lallemand D, Aubin ML, Vignaud J, LavalJeantet M (1988) Separation of diffusion and perfusion in intravoxel incoherent motion MR imaging. Radiology 168:497-505

8. Le Bihan D, Breton E, Lallemand D, Grenier P, Cabanis E, LavalJeantet M (1986) MR imaging of intravoxel incoherent motions: application to diffusion and perfusion in neurologic disorders. Radiology 161:401-407

9. Luciani A, Vignaud A, Cavet M, Nhieu JT, Mallat A, Ruel L, Laurent A, Deux JF, Brugieres P, Rahmouni A (2008) Liver cirrhosis: intravoxel incoherent motion MR imaging-pilot study. Radiology 249:891-899

10. Shimada K, Isoda H, Hirokawa Y, Arizono S, Shibata T, Togashi K (2010) Comparison of gadolinium-EOB-DTPA-enhanced and diffusion-weighted liver MRI for detection of small hepatic metastases. Eur Radiol 20(11):2690-8 
11. Taouli B, Koh DM (2010) Diffusion-weighted MR imaging of the liver. Radiology 254:47-66

12. Vandecaveye V, De Keyzer F, Verslype C, Op de Beeck K, Komuta M, Topal B, Roebben I, Bielen D, Roskams T, Nevens F, Dymarkowski S (2009) Diffusion-weighted MRI provides additional value to conventional dynamic contrast-enhanced MRI for detection of hepatocellular carcinoma. Eur Radiol 19:24562466
13. Wang H, Li J, Chen F, De Keyzer F, Yu J, Feng Y, Nuyts J, Marchal G, Ni Y (2010) Morphological, functional and metabolic imaging biomarkers: assessment of vascular-disrupting effect on rodent liver tumours. Eur Radiol 20:2013-2026

14. Yamada I, Aung W, Himeno Y, Nakagawa T, Shibuya H (1999) Diffusion coefficients in abdominal organs and hepatic lesions: evaluation with intravoxel incoherent motion echo-planar MR imaging. Radiology 210:617-623 